\title{
Estrogens Administration in Female Transsexuals Augmented Delta and Rem Sleep Stages after Six Months of Treatment
}

\author{
Evalinda Barrón-Velázquez ${ }^{1}$, Daniel Santana ${ }^{2}$, Pamela Espinosa ${ }^{1}$ and Rafael J. Salin-Pascual ${ }^{1, *}$ \\ ${ }^{I}$ Department of Psychiatry, School of Medicine, National University Autonomous of Mexico, Mexico \\ ${ }^{2}$ General Hospital of Mexico, Health Ministry, Mexico
}

\begin{abstract}
The transsexual condition (TS) (Gender Dysphoria DSM-5) people have a marked incongruence between the gender they have been assigned at birth and their experienced/expressed gender. There are variations in brain structures that express reproductive behavior mainly in the hypothalamus. Also, there are sleep differences between non-transsexual female and male. This gender dimorphism in the sleep regulation is due to anatomical changes previously reported in the hypothalamus, which would suggest that it could also find significant differences in sleep architecture in transsexual persons, before and after hormonal treatment with estrogens.
\end{abstract}

Six persons diagnosed with gender identity disorder according to DSM IV-TR, were studied. Also 16 healthy volunteers men $(n=6)$ and women $(n=10)$ were included. A polysomnography base study in which a baseline night recording as well as at 3 and 6 months was performed, with a portable polysomnographic system, for MTF transsexuals. Healthy volunteers were studied only one baseline night. Hormone therapy was administered as conjugated estrogens $0.625 \mathrm{mg} / \mathrm{d}$. (PremarinPfizer), within six months of the present protocol, there were measurements of hormone levels at baseline, three to six months.

A descriptive analysis of sexual hormones in plasma, before hormone treatment and sleep variables were performed. They were within normal distribution. The Kolmogorov Smirnov test to asses normality within sleep variables showed normal distribution, so that parametric tests for comparison of those variables could be made.

The main significant differences between male and female controls were that the second group had short sleep latency and higher REM sleep time and REM sleep percentage. Sleep variables between female controls and MTF after six months of estrogens had differences in sleep stages 2, 3 and REM.

Then a correlation analysis between the hormone levels at different time of their administration and percentages for each of the sleep variables at baseline, 3 and 6 months were performed and it was found that the proportion of N2 is linked to changes in the levels of luteinizing hormone having a Person correlation coefficient of - 0.484. Finally, an ANOVA and post hoc analysis was performed to see the difference between sleep variables in transsexual subjects at baseline and at 6 months vs. non-transsexual women and men.

Conclusions: The main findings of the present study were that after six months of treatment with estrogens in MTF transsexuals there was an increase in sleep stages 2, 3 and REM, comparing with female controls, and that correlates with luteinizing hormone.

Keywords: Brain plasticity, Gender-Estrogens, Sleep, Transsexuals.

\section{INTRODUCTION}

The transsexual condition (TS) (Gender Dysphoria DSM5) $[1,2]$ is conceptualized as follows: They have a marked incongruence between the gender they have been assigned at birth and their experienced/expressed gender. That is, they perceives as women in a men's body or vice versa. The first condition is known as a transsexual woman (Male to Female MTF) and the second as a transsexual man (Female to Male FTM). These people expressed their incongruence from an

*Address correspondence to this author at the School of Medicine, National University Autonomous of Mexico, Hidalgo 187 casa 7, Barrio del Niño Jesus, Tlalpan, Mexico City 14080, México; E-mails: rafasalin@yahoo.com, rafasalinpas@gmail.com early age, as an inconsistency with their appearance and subjective gender, and the willingness to modify that through surgical and/or hormonal methods, with the aim to make them as coherent as possible to their desired gender [3].

The incidence and prevalence are little known in most countries. It has been reported a huge variability globally, that oscillate: $1: 24,000$ to $1: 100,000$ for transsexual women; and from 1:100,000 to 1:400,000 for transsexuals men $[4,5]$.

Inquiries about genetic components in transsexuality, suggest that in infants, atypical gender identity was well supported [6]. In the sixth week of intrauterine development due to the activity of the gonad related to sex chromosomes, indicated by XX / XY, ovaries or testes, take control of fetal hormone levels, and participate in the feminization or 
masculinization of the fetus [7]. Correlation was detected between DNA markers on the $\mathrm{X}$ chromosome, in a region called $\mathrm{Xq} 28$ and the presence of male homosexuality; however, this information has not been replicated [6].

The sexual differentiation of the brain occurs later in fetal development, and therefore occurs independently of sexualgenital differentiation. This brain differentiation has been estimated that occurs around the fourteenth week of development. Prior to this week, the fetal brains are all female, by the influences of maternal hormones. If fetal sex glands, do not perform their function, or if there are obstacles to the arrival of hormones to their receptors, or if they work improperly that would explain some about transsexuality as well as sexual orientation later on life. That confirms that the sex-differentiation and sexual orientation event occurs at intrauterine level and there are no evidence of post-natal processes that may be a causal agents of gender or sexual diversity [8].

There are some hypothesis about that human condition, however, is not known at what time occur throughout pregnancy and what are the factors that determine it. Changes in the structures of the sex-gender diversity are well supported by biology and genetics evidences. There are variations in brain structures that express reproductive behavior mainly in the hypothalamus [9-11].

Veale et al. [12] reviewed previous studies of this condition. Reported that there is evidence of a genetic component of sex-gender diversity based on twin studies and other individuals within the concordance of families studied that have specifically examined certain group of genes. Also report evidence of prenatal androgen levels, which correlate with gender diversity, and fine morphological changes, such as studies of finger length (2D: 4D) between transgender and non-transgender individuals. In addition, there is evidence that transsexuals have parts of brain structures, that resembles to the genre that feel they belong to $(\mathrm{Vg}$, not transsexual women and transsexual women), there is a greater probability of being non-right-handed, more likely to report child abuse, and to have a greater number of older brothers [10, 13].

The high number of older brothers in transsexual women, has also been observed in homosexual men. For this, it is proposed that each male fetus creates progressive immunization in the presence of antigen to the testosterone receptors so in the future pregnancies if the fetus is male, there would be less masculinization in certain brain areas [10]. That would rize some explanationn about transsexuals and homosexuals as part of some evolutive strategies [14].

Whereas some brain area involved in the gender identity are found in the hypothalamus, that same structure that is part of sleep regulatory areas and a high involvement in circadian rhythms as well, it is important to study its relevance in transsexual patients, since it has been demonstrated a hormonal influence on the sleep-wake cycle in female and gender differences as well [6]. There were reported differences in sleep patterns between men and not transsexual women. In adulthood, women have a better sleep quality overall (shorter sleep onset latency, higher efficiency) compared with men; however, complaints of insomnia and daytime sleepiness are more common in women ( $58 \%$ vs. $43 \%$ in men). Differences in quality of sleep and the prevalence of sleep disorders in women, are due in part because in woman, there are greater effect of hormonal changes, which are supposed to have an impact on some type of sleep disturbance as insomnia or hypersomnia $[15,16]$.

In a study in which was evaluating gender differences in sleep patterns, the effects of menopause and hormone therapy, it was found that women compared with men, had a significantly higher percentage of total sleep time, lower percentage sleep stage 1, higher delta percentage before menopause. Moreover, in menopause with no hormone treatment, was associated with prolonged sleep latency and decreased sleep depth. Finally, young, healthy women compared with men, matched for age, experience fewer sleep disturbances when subjected to stress, as assessed by minor changes in sleep time and the percentage of phase 1 sleep $[17,18]$.

As we can see, the gonadal hormones exert a beneficial effect on women's sleep. This gender dimorphism in the sleep regulation is due to anatomical changes previously reported in the hypothalamus, which would suggest that it could also find significant differences in sleep architecture in transsexual persons, before and after hormonal treatment with estrogens.

The main objective of this study was to see if there are changes in sleep architecture, assessed objectively by polysomnographic recordings, before, and after three and six months of estrogen administration in transsexual woman.

\section{MATERIAL AND METHODS}

This is a prolective, prospective and longitudinal clinical study type, with quasi-experimental assignment, aimed to transsexual women with conjugated estrogens administered for a period of six months, and with the evaluation of the effect on these hormones on sleep architecture.

Recruitment procedure: People diagnosed with gender identity disorder according to DSM IV-TR. These were transsexual women (MTF), over 18 years old, naive to hormonal treatment or that they had not taken hormones, at least one year prior to the study. TS women were excluded if they have the following clinical conditions: endocrine diseases, severe hypertension, thrombophlebitis, liver dysfunction, comorbid psychiatric disorders, those that should receive drug therapy for medical or psychiatric conditions or sleep disorders.

It also included 16 healthy volunteers men $(n=6)$ and women $(n=10)$. To whom are medical and psychiatrically evaluated to validate its status of healthy volunteers. The ages of these volunteers were similar to those of transsexual women

Both transgender and healthy volunteers were explained in detail the protocol, plus they were given a letter as informed consent, that once answered their questions and decided to participate in the study, the informed consent was 
Table 1. Variables in MTF transsexuals related to age of awareness and gestational order.

\begin{tabular}{|c|c|c|c|}
\hline & $\mathbf{N}$ & $\mathbf{X}$ & SD \\
\hline Age (years) & 6 & 25.17 & \pm 9.390 \\
\hline Age of Awareness of Gender Condition (years) & 6 & 5.33 & \pm 1.862 \\
\hline Gestational Order & 6 & 1.83 & \pm 0.753 \\
\hline
\end{tabular}

signed. The protocol on which this study is part of, has been approved by the Ethical and Scientific Committees of the School of Medicine of the National Autonomous University of Mexico and the General Hospital of Mexico, Health Ministry.

\section{METHODS}

A polysomnography base study in which a baseline night recording as well as at 3 and 6 months was performed, with a portable polysomnographic system: Aura GRASS consisting of 55 Total Channels: 12 analog inputs, 26 channels (10 left, 9 right, 2 EOG 5 EMG and reference 2) input impedance: 1.66 MOhm 3.33 per electrode differential, Bandwidth: 0.32 $\mathrm{Hz}$ to $106 \mathrm{~Hz} 0.32 \mathrm{~Hz}$ to $106 \mathrm{~Hz}$, signal input range: $\pm 3.3 \mathrm{mV} \pm 3.3 \mathrm{mV}$, Frequency sampling: $2000 \mathrm{~Hz} 2000 \mathrm{~Hz}$ cutaneous electrodes were applied according to the distribution of electrodes of the American Academy of Sleep and the International System for 10-20 electroencephalographic recordings, using a limited number of channels: $\mathrm{C} 4$ and C3-A1-A1. The classification of sleep stages was performed according to standard criteria of Rechtschaffen and Kales 1968 [19], by a sleep medicine specialist who was blind to the order of assignment of the studies and whether corresponded to a transsexual person or a healthy volunteers.

Subjects were studied with all-night polysomnographic recordings (electroencephalogram, oculogram, electromyogram and EKG) Polysomnography studies began at 22:00 $\mathrm{Hr}$ and ended eight hours later $(06: 00 \mathrm{Hr})$. Sleep onset (i.e., Sleep Latency) was defined as the first period of eight continuous minutes of sleep after lights-out; awake time was defined as the minutes awake after sleep onset; REM sleep latency was defined as the time from sleep onset, to the first two periods of REM sleep that lasted more than one minute. Variables reflecting sleep architecture included time in each sleep stage (i.e., I, II, Delta and REM sleep).

Hormone therapy was the administration of conjugated estrogens $0.625 \mathrm{mg} / \mathrm{d}$. (Premarin-Pfizer), within six months of the present protocol, there were measurements of hormone levels at baseline, three to six months.

Statistical analysis: a Kolmogorov-Smirnov test was used to check normality, then a general linear model for repeated measures to compare each of the variables of baseline sleep, at three and six months; analysis and Pearson correlation between the levels of hormones, sleep variables and ANOVA test for sleep variables among transsexuals subjects and control subjects was also performed.

\section{RESULTS}

Forty transexual woman were evaluated before enter the study. After screening six transsexual women were accepted. Some varibles related to transsexuality features for MTF transsexuals are shown in Table 1. They were in the second decade of life, with average high school education, and an age of realizing their generic inconsistency before 12 years old. Six of them had older brothers. Female controls' age were $28.0 \pm 10.10$ year old. Male controls' age were $22.3 \pm$ 4.8 years old.

A descriptive analysis of sexual hormones in plasma, before hormone treatment and sleep variables were performed. They were within normal distribution. The Kolmogorov Smirnov test for to asses normality within sleep variables showed normal distribution, so that parametric tests for comparison of those variables were made.

Table 2, shows sleep variable between controls groups. The main significant differences between male and female controls were that the second group had short sleep latency and higher REM sleep time and REM sleep percentage.

Sleep variables between female controls and MTF after six months of estrogens had differences in sleep stages 2, 3 and REM sleep (Table 3).

A general linear model for repeated measures was performed to see whether there were differences between baseline levels, 3 and 6 month, between hormone levels and sleep variables. Statistically significant baseline levels of estradiol and prolactin to 24 weeks of treatment were found increased, however no differences were observed between testosterone, follicle stimulating hormone, luteinizing hormone and progesterone in transsexual women.

The sleep variables were statistically significant in $\mathrm{N} 1$ and $\mathrm{N} 2$ between and the end of the study, although the effect size for the percentage of N1 was small, that can be seen in Fig. (1) that the differences in the percentage of sleep stage $\mathrm{N} 2$ decreased significantly a long the study $(\mathrm{p}<0.005)$. No other significant differences in the sleep variables were found.

Then a correlation analysis between the hormone levels at differente time of their administration and percentages for each of sleep variables an baseline, 3 and 6 months were performed and it was found that the proportion of $\mathrm{N} 2$ is linked to changes in the levels of luteinizing hormone having a Person correlation coefficient of - 0.484. Finally, an ANOVA and post hoc analysis (Table 4) was performed to see the difference between sleep variables in transsexual subjects at baseline and at 6 months vs. non-transsexual women and men. 
Table 2. Female vs. male control groups.

\begin{tabular}{|c|c|c|c|}
\hline Group & FEM & Male & t Student \\
\hline TST (min) & $442.3 \pm 31.8$ & $423 \pm 11.4$ & 0.179 \\
\hline $\mathrm{SL}(\mathrm{min})$ & $9.0 \pm 5.04$ & $22.1 \pm 9.2$ & 0.002 \\
\hline REM L. (min) & $106.05 \pm 46.3$ & $107.25 \pm 62.02$ & 0.965 \\
\hline Sleep Efc \% & $90.33 \pm 6.6$ & $85.92 \pm 2.7$ & 0.148 \\
\hline Stage N1 (min) & $23.4 \pm 18.35$ & $37.41 \pm 19.7$ & 0.171 \\
\hline Stage N2 (min) & $224.35 \pm 28.4$ & $223.66 \pm 30.55$ & 0.964 \\
\hline Stage N3 (min) & $97 \pm 17.1$ & $96.33 \pm 28.88$ & 0.962 \\
\hline Stage REM (min) & $97.55 \pm 25.1$ & $65.58 \pm 11.38$ & 0.011 \\
\hline Stage $1(\%)$ & $6.19 \pm 4.3$ & $9.414 \pm 4.65$ & 0.153 \\
\hline Stage $2(\%)$ & $49.52 \pm 4.3$ & $51.55 \pm 4.65$ & 0.461 \\
\hline Stage $3(\%)$ & $20.87 \pm 4.2$ & $21.4 \pm 7.78$ & 0.869 \\
\hline REM Sleep (\%) & $21.51 \pm 5.1$ & $14.78 \pm 2.64$ & 0.008 \\
\hline REM activity & $290.2 \pm 20.39$ & $242.0 \pm 49.8$ & 0.165 \\
\hline REM density & $3.13 \pm 1.36$ & $3.52 \pm 0.53$ & 0.518 \\
\hline REM average & $22.64 \pm 7.06$ & $25.11 \pm 12.7$ & 0.631 \\
\hline
\end{tabular}

Female controls $v s$. Transsexual women and Male controls $v s$. Transsexual women were not differents in sleep variables and baseline night (data are not shown).

Table 3. Sleep stages female control vs. MTF six months.

\begin{tabular}{|c|c|c|c|}
\hline Sleep Variables & Female Controls & MTF After Six Months & Student t Test \\
\hline \hline Total Sleep Time (TST): & $442.3+31.8$ & $396.08+56.03$ & $\mathrm{~ns}$ \\
\hline Sleep Latency: & $9+5.0$ & $10.58+4.6$ & $\mathrm{~ns}$ \\
\hline Stage R Latency: & $106.05+46.31$ & $109.16+37.49$ & $\mathrm{~ns}$ \\
\hline Sleep Stage 1 & $23.4+18.3$ & $18.58+10.22$ & 0.0005 \\
\hline Sleep Stage 2 & $224.35+28.39$ & $164.75+22.6$ & 0.02 \\
\hline Sleep Stage 3 & $95.0+17.10$ & $139.83+47.68$ & 0.03 \\
\hline REM sleep & $97.55+25.10$ & $62.16+34.8$ & \\
\hline
\end{tabular}

Tabla 4. Anova for sleeping and hormone levels.

\begin{tabular}{|c|c|c|c|}
\hline Sleep Variable & Time of Hormone Treatment & Group Effect \\
\hline \hline N1\% & MTF baseline & MTF6 months & Female Control \\
& & Male Control &, 47 \\
& & MTF baseline &, 87 \\
\hline N2\% & MTF6 months & Female Control &, 009 \\
& & Male Control &, 01 \\
\hline
\end{tabular}


Table 4. contd...

\begin{tabular}{|c|c|c|c|}
\hline Sleep Variable & Time of Hormone Treatment & Group Effect & Sig \\
\hline N3 \% & MTF6 months & $\begin{array}{c}\text { MTF baseline } \\
\text { Female Control } \\
\text { Male Control }\end{array}$ & $\begin{array}{l}, 01 \\
, 020 \\
, 003\end{array}$ \\
\hline REM sleep \% & MTF baseline & $\begin{array}{c}\text { MTF6 months } \\
\text { Female Control } \\
\text { Male Control }\end{array}$ & $\begin{array}{l}, 60 \\
, 08 \\
, 17\end{array}$ \\
\hline Sleep Efficiency & MTF baseline & $\begin{array}{c}\text { MTF6 months } \\
\text { Female Control } \\
\text { Male Control }\end{array}$ & $\begin{array}{l}, 08 \\
, 44 \\
, 05\end{array}$ \\
\hline $\begin{array}{c}\text { REM sleep } \\
\text { Episodes }\end{array}$ & MTF 6 months & $\begin{array}{c}\text { MTF baseline } \\
\text { Female Control } \\
\text { Male Control }\end{array}$ & $\begin{array}{r}, 002 \\
, 003 \\
, 003\end{array}$ \\
\hline
\end{tabular}

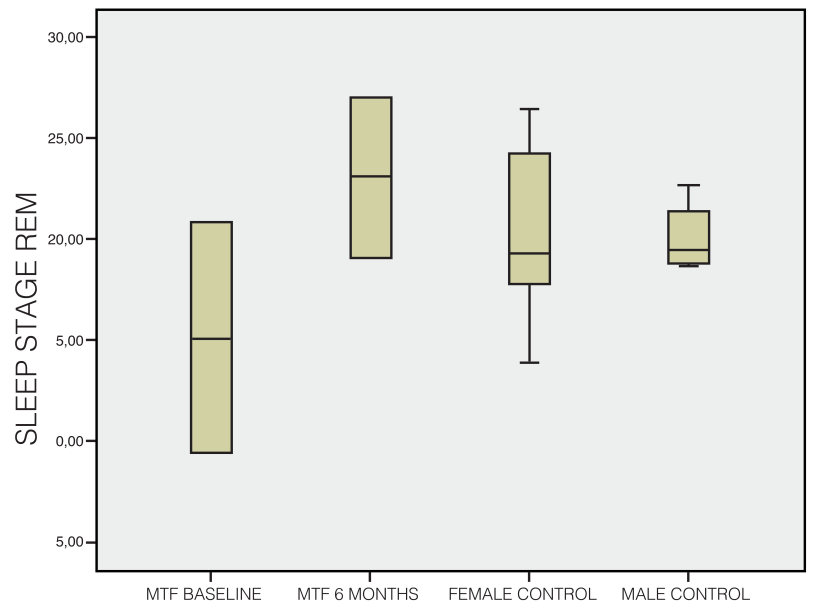

Fig. (1). Percentage of sleep stage 2 in MTF and control groups.

A significant reduction in the percentage of sleep stage $\mathrm{N} 2$ in transsexual women at 6th months of hormone reassignment therapy was observed, No other differences were found among the other two groups (Fig. 1).

Differences in sleep stage 3 , between transsexual women with 6 months of hormone therapy, and the other two groups were found significant (see Fig. 2).

Also, differences in REM sleep percentage were found between transsexual women at baseline and after six month with hormonal therapy. (See Fig. 3).

Other sleep variables were not with significant differences. No side effects that stop hormonal treatment were reported for the MTF persons in the present study.

\section{DISCUSSION}

The main findings of the present study were that after six months of treatment with crosshormone in MTF transsexuals there was an increase in sleep stages 2, 3 and REM, comparing with female controls.

Gender differences between non-transsexuals male and female have been reported previously [20]. In the present study a baseline comparison between male and female control, showed that overall female had a shorter sleep latency), higer amount of REM sleep and non significative increase in sleep efficiency percentage.

By the sixth month of hormones administration MTF had a significant increase in the overall percentage of sleep stages that correlates positively with the estrogens levels. Sleep questionaries and expontaneus verbal reports of them supported the feeling of well being as result of better sleep quality.

Menopausal women had an increase of sleep problems and that had been explained by hormonal changes [21, 22]. Estrogen treatment after menopause can help to restore the normal sleep electroencephalogram pattern, which in turn 


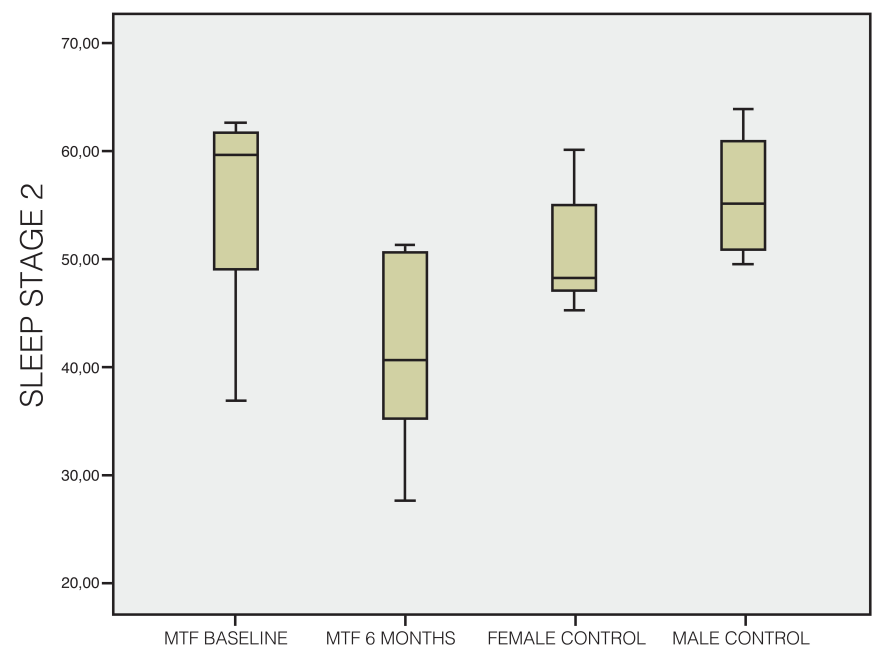

Fig. (2). Percentage of sleep stage $3 \mathrm{MTF}$ and control groups.

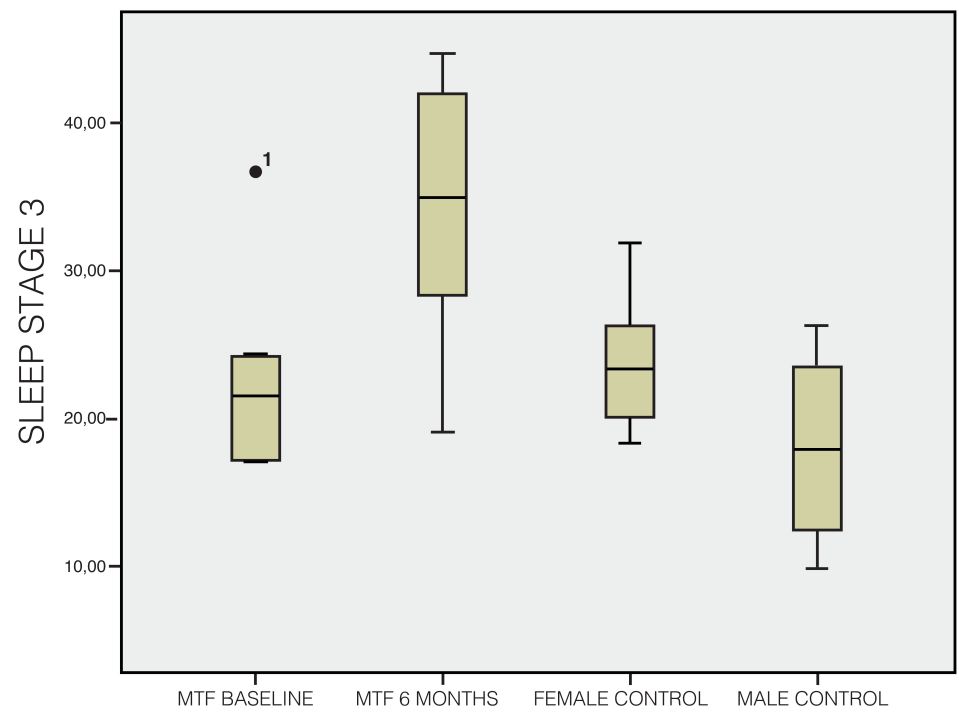

Fig. (3). REM sleep percentage in MTF and control groups.

might contribute to improved cognitive functioning [23]. Similar results after estrogens administration were found for the percentages of total sleep time and of total non-rapid eye movement sleep in menopausal women [24].

The present finding in transsexual women support the role of estrogens in sleep that was even increased than control women. In a previous study Kunzel et al examined seven male to female transsexual patients (age range 31-44 years, mean+/-SD 35.9+/-4.2 years). The first examination was performed before and the second about three months after initiation of cross-gender hormone therapy with a dose of $80-100 \mathrm{mg}$ estrogen applied every two weeks.
Additionally patients were treated with a starting dose of the anti-androgen cyproteronacetate of $100 \mathrm{mg} / \mathrm{day}$ and after about 6 weeks with a maintenance therapy of $50 \mathrm{mg} / \mathrm{d}$ in order to suppress androgenic effects. They concluded that the administration of estrogen and anti-androgens in male to female transsexual patients had only a small influence on sleep EEG, with an increase in the duration of shallow sleep [25].

The MTF transsexual of the present study was given only estrogens in the same amount $(0.625 \mathrm{mg} /$ day $)$ during the six months. That represent, as far of our knowledge the first report of such effect on sleep in relative naïve person to 
estrogens, with biological phenotype od sexual chromosomes XY.

The estrogens administration in transsexual person is part of their treatment for to make coherent their mind with their bodies, but at the same time is a natural model of the effects of such hormones in several functions. As has been mentioned before, one of the areas of transsexual brain that are different between the rests of humans is hypothalamus, that also modulates the sleep wake cycle [26].

Onset Age (OA) has a discriminative value for transsexual developments and it would appear that retrospective diagnosis of Gender Identity disorder criteria as a valid method of assessment. Differences in OA and sex ratio exist between European countries [8]. That has been mentioned in DSM-5 [2] as a subdivision of Onset of cross gender behavior is usually between ages 2 t o 4 years. Early OA usually is associated with sexual orientation toward masculine people and with androgenic attitudes, which could help in some cases to a grater acceptance in the family.

Age of awareness in the sample of this study was around five years old, and also they had older brothers. Both conditions had been speculated to give a different expression of their transsexual condition. Two bio-demographic variables - birth order and sibling sex ratio - have been examined in several Western samples of homosexual transsexual men [13]. The results have consistently shown that homosexual transsexuals have a later birth order and come with an excess of brothers to sisters; the excess of brothers has been largely driven by the number of older brothers and hence has been termed the fraternal birth order effect. About this there are some hypothesis that the excess of older brothers could induce some biological responses in the mother like antibodies toward testosterone receptors that in the development brain may produce a different pattern of hypothalamus neuronal circuits, that is that male gender identity might be partly mediated through the androgen receptors [27].

The sleep findings of this study may be explained related to some brain changes as result of estrogens administration. There are some studies on the effects of treatments on brain cortical thickness (CTh) of FTM and MTF persons. After testosterone treatment, FTM showed increases of CTh bilaterally in the post central gyrus and unilaterally in the inferior parietal, lingual, pericalcarine, and supramarginal areas of the left hemisphere and the rostral middle frontal and the cuneus region of the right hemisphere. Estrogens and ant androgens therapy in MTF is associated to a decrease in the CTh that consequently induces an enlargement of the ventricular system [28]. Are these changes in cortical areas related to the sleep findings in the present study? That is new research questions that need new approaches for to be solved.

The main limitations of the present study are the low number of FTM transsexual persons, and that the time of follow-up. However, there are some methodological issues that difficult to resolve that in the short therm. Some of the well being of hormonal reassignment, other than the external coherent aspect could be also the repairing effect of an increase amount of sleep.

\section{CONFLICT OF INTEREST}

The authors confirm that this article content has no conflicts of interest.

\section{ACKNOWLEDGEMENTS}

Declared none.

\section{REFERENCES}

[1] Heylens G, Elaut E, Kreukels BP, et al. Psychiatric characteristics in transsexual individuals: multicentre study in four European countries. Br J Psychiatr 2014; 204(2): 151-6.

[2] Association AP. Diagnostic and Statistical manual of mental disorders $5^{\text {th }}$ ed.: American Psychiatric Association Press 2013.

[3] Simon L, Zsolt U, Fogd D, Czobor P. Dysfunctional core beliefs, perceived parenting behavior and psychopathology in gender identity disorder: a comparison of male-to-female, female-to-male transsexual and nontranssexual control subjects. J Beh Ther Exp Psychiatr 2011; 42(1): 38-45.

[4] Duisin D, Nikolic-Balkoski G, Batinic B. Sociodemographic profile of transsexual patients. Psychiatr Danub 2009; 21(2): 220-3.

[5] Gomez-Gil E, Trilla A, Salamero M, Godas T, Valdes M. Sociodemographic, clinical, and psychiatric characteristics of transsexuals from Spain. Arch Sex Behav 2009; 38(3): 378-92.

[6] Lombardo F, Toselli L, Grassetti D, et al. Hormone and genetic study in male to female transsexual patients. J Endocrinol Invest 2013; 36(8): 550-7.

[7] Fuss J, Biedermann SV, Stalla GK, Auer MK. On the quest for a biomechanism of transsexualism: is there a role for BDNF? J Psychiat Res 2013; 47(12): 2015-7.

[8] Nieder TO, Herff M, Cerwenka S, et al. Age of onset and sexual orientation in transsexual males and females. J Sex Med 2011; 8(3): 783-91.

[9] Friedman G. The effects of estrogen on short-term memory in genetic men. J Am Med Direct Ass 2000; 1(1): 4-7.

[10] Green R. Birth order and ratio of brothers to sisters in transsexuals. Psychol Med 2000 30(4): 789-95.

[11] Green R, Keverne EB. The disparate maternal aunt-uncle ratio in male transsexuals: an explanation invoking genomic imprinting. J Theor Biol 2000; 202(1): 55-63.

[12] Veale JF. Prevalence of transsexualism among New Zealand passport holders. Aus N Z J Psychiatr 2008; 42(10): 887-9.

[13] Zucker KJ, Blanchard R, Kim TS, Pae CU, Lee C. Birth order and sibling sex ratio in homosexual transsexual South Korean men: Effects of the male-preference stopping rule. Psychiat Clin Neurosci 2007; 61(5): 529-33.

[14] Bailey NW, Zuk M. Same-sex sexual behavior and evolution. Trends Ecol Evol 2009; 24(8): 439-46.

[15] Goel N, Kim H, Lao RP. Gender differences in polysomnographic sleep in young healthy sleepers. Chronobiol Int 2005; 22(5): 905 15 .

[16] Voderholzer U, Al-Shajlawi A, Weske G, Feige B, Riemann D. Are there gender differences in objective and subjective sleep measures? A study of insomniacs and healthy controls. Depress Anx 2003; 17(3): 162-72.

[17] Lampio L, Polo-Kantola P, Polo O, Kauko T, Aittokallio J, Saaresranta T. Sleep in midlife women: effects of menopause, vasomotor symptoms, and depressive symptoms. Menopause 2014; 21(11): 1217-24.

[18] Landis CA, Moe KE. Sleep and menopause. Nursing Clin North Am 2004; 39(1): 97-115.

[19] Rechtschaffen A, Kales A. A manual of standardized terminology, techniques and scoring system for sleep stages of human subjects. Arch Gen Psychiatry 1969; 20(2): 246-7.

[20] Moline ML, Broch L, Zak R, Gross V. Sleep in women across the life cycle from adulthood through menopause. Sleep Med Rev 2003; 7(2): 155-77. 
[21] Pien GW, Sammel MD, Freeman EW, Lin H, DeBlasis TL. Predictors of sleep quality in women in the menopausal transition. Sleep 2008; 31(7): 991-9.

[22] Murphy PJ, Campbell SS. Sex hormones, sleep, and core body temperature in older postmenopausal women. Sleep 2007; 30(12): 1788-94.

[23] Antonijevic IA, Stalla GK, Steiger A. Modulation of the sleep electroencephalogram by estrogen replacement in postmenopausal women. Am J Obstet Gynecol 2000; 182(2): 277-82.

[24] Manber R, Kuo TF, Cataldo N, Colrain IM. The effects of hormone replacement therapy on sleep-disordered breathing in postmenopausal women: a pilot study. Sleep 2003; 26(2): 163-8.
[25] Kunzel HE, Murck H, Stalla GK, Steiger A. Changes in the sleep electroencephalogram (EEG) during male to female transgender therapy. Psychoneuroendocrinology 2011; 36(7): 1005-9.

[26] Salin-Pascual R, Gerashchenko D, Greco M, Blanco-Centurion C, Shiromani PJ. Hypothalamic regulation of sleep. Neuropsychol Pharmacol 2001; 25: S21-7.

[27] Hare L, Bernard P, Sanchez FJ, Baird PN, Vilain E, Kennedy T, et al. Androgen receptor repeat length polymorphism associated with male-to-female transsexualism. Biol Psychiatry 2009; 65(1): 93-6.

[28] Zubiaurre-Elorza L, Junque C, Gomez-Gil E, Guillamon A. Effects of cross-sex hormone treatment on cortical thickness in transsexual individuals. J Sex Med 2014; 11(5): 1248-61.

(C) Barrón-Velázquez et al.; Licensee Bentham Open.

This is an open access article licensed under the terms of the Creative Commons Attribution Non-Commercial License (http://creativecommons.org/licenses/by$\mathrm{nc} / 3.0 /$ ), which permits unrestricted, non-commercial use, distribution and reproduction in any medium, provided the work is properly cited. 